ఠ

\title{
Benefit-risk assessment of new and emerging treatments for hepatitis $C$ : focus on simeprevir and sofosbuvir
}

REVIEW

John N Gaetano

Section of Gastroenterology, Hepatology and Nutrition, Department of Medicine, University of Chicago Hospitals, Chicago, IL, USA
This article was published in the following Dove Press journal:

Drug, Healthcare and Patient Safety

31 March 2014

Number of times this article has been viewed
Correspondence: John N Gaetano Section of Gastroenterology, Hepatology and Nutrition, Department of Medicine, University of Chicago Hospitals,

584I S Maryland Ave, MC 4076, Chicago, IL 60637, USA

Tel +l 7737028283

Email john.gaetano@uchospitals.edu
Abstract: Greater understanding of the hepatitis $\mathrm{C}$ virus (HCV) genome and life cycle of the HCV virion allows for new targets for therapy that directly act on the viral machinery to inhibit replication. Numerous direct-acting antivirals are in development, and four have been brought to market. Simeprevir, a second-generation protease inhibitor, has been approved for $\mathrm{HCV}$ genotype 1 patients in combination with pegylated interferon- $\alpha$ and ribavirin. Sofosbuvir, a novel nucleotide analog, has pangenotypic coverage and has been approved for HCV genotype 1 patients with ribavirin and pegylated interferon- $\alpha$. For HCV genotypes 2 and 3, an all-oral regimen of sofosbuvir with ribavirin has become the new gold standard for treatment. The efficacy and safety for these two novel therapies among various subpopulations of those infected with chronic hepatitis $\mathrm{C}$ are discussed in the following review. In addition, off-label and future therapeutic regimens are addressed, as well as the concerns about cost of current and future therapies.

Keywords: liver disease, treatment-naïve and treatment experienced, HCV genotype

\section{Introduction}

Since 1982, when hepatitis C (HCV) infection became a reportable virus, it has become the most common blood-borne infection in the United States and worldwide. In the United States, estimates of prevalence range from $1.3 \%$ to $2.0 \%$, with more than 5.2 million people living with chronic HCV. ${ }^{1}$ Whereas new cases of HCV infection have drastically declined over the past three decades due to awareness and public health policies, underdiagnosis and undertreatment have contributed to a continuing challenge in combating chronic $\mathrm{HCV}^{2}$ In addition, $\mathrm{HCV}$ infection largely affects an aging population, driving liver disease to become the twelfth leading cause of death in the United States and the fourth leading cause of death in persons aged 45-64 years. Even these numbers, although significant, likely underestimate the mortality from liver disease in the US. ${ }^{3}$ By many estimates, the burden of liver disease due to chronic HCV will dramatically increase over the next two decades until a significant majority of individuals are cured of their infection. The goal of treatment is to reduce the high morbidity and mortality associated with the natural history of chronic infection particularly, the development of hepatic fibrosis, cirrhosis, and it complications, such as portal hypertension and hepatocellular carcinoma.

The landscape of HCV therapy has dramatically changed since initial trials with interferon monotherapy in the 1980s. Clinical trials with interferon followed by addition of ribavirin (RBV) and use of pegylated interferon- $\alpha$ (PEG) showed steadily improved sustained virologic response (SVR) rates in the 1990s and early 2000s. 
These therapeutic trials were aided by advances in molecular diagnostics that allowed for accurate assessment of virologic response. Even though progress was tangible, with SVR rates steadily improving from less than $20 \%$ to nearly $70 \%$ in select patients, wide application of these therapies was greatly limited due to treatment contraindications, side effects, and low SVR rates in individuals with advanced fibrosis and prior treatment failures. ${ }^{4,5}$

\section{The era of the direct-acting antivirals}

Greater understanding of the HCV genome and the life cycle of the HCV virion has revealed new targets for therapy that act directly on the viral machinery to inhibit replication. Consequently, treatment of HCV has now evolved beyond stimulation of the host immune system and nonspecific targeting of viral replication. Coined direct-acting antivirals (DAAs), these new drugs include a diverse class of compounds that take advantage of this understanding of the $\mathrm{HCV}$ genome and lifecycle.

Among the proteins that are encoded in the HCV RNA, there are structural and nonstructural (NS) proteins. Among NS proteins, the serine-like protease encoded in the NS3 region and the RNA-dependent RNA polymerase encoded in the NS5B region have been targets for the DAA compounds. ${ }^{6,7}$

An NS3 serine protease and a cofactor, NS4A, allow for posttranslational cleavage of the 3,000 amino acid polyprotein produced from HCV RNA and host ribosomes. Once cleaved, this polyprotein forms four structural and six NS proteins key in viral RNA replication and new viral particle assembly. NS3/4A complex is the direct target of the firstapproved, or "first-generation," protease inhibitors. ${ }^{8}$

Boceprevir (BOC) and telaprevir (TVR), the first-generation protease inhibitors, combined with PEG/RBV brought improvement in overall SVR rates from approximately $40 \%$ to $60 \%$ in treatment naïve individuals, while treatment duration ranged from 24-48 weeks, depending on HCV RNA decline throughout therapy. Cirrhotic patients required 48 weeks of therapy regardless of viral response. In addition to the common side effects seen with PEG/RBV, BOC and TVR compounded treatment difficulty with unique side effects of their own. ${ }^{9-12}$

With BOC, anemia, neutropenia, and dysgeusia were most common side effects, whereas anemia, skin rash, and anorectal symptoms were more frequent with TVR. Anemia was recognized as a common adverse event, occurring in about $50 \%$ of individuals treated with BOC and $40 \%$ of those treated with TVR. Anemia frequently led to the use of erythropoietin-stimulating agents, blood transfusions, or treatment discontinuation with subsequent treatment failure in a significant number of individuals. In addition, TVR-based therapy resulted in a more than $20 \%$ higher incidence of rash and pruritus compared to placebo. Although 90\% of rash events related to TVR were mild or moderate and did not progress, $6 \%$ of all patients required treatment discontinuation. Gastrointestinal symptoms are another common group of adverse events for patients treated with BOC or TVR. Dysgeusia was present in $35 \%-44 \%$ of patients treated with BOC, and anorectal symptoms such as anal pruritus and rectal burning were seen in $26 \%$ of patients treated with TVR. ${ }^{13}$

\section{The evolving standard of care}

Numerous protease inhibitors beyond TVR and BOC are in development. These new drugs have several factors that make them advantageous, including high potency, multigenotypic coverage, and an intermediate-to-high barrier to resistance. Some second-wave protease inhibitors are being tested in combination with ritonavir, a CYP3A inhibitor, to reduce overall protease inhibitor exposure while maintaining potent antiviral activity and attempting to minimize toxicities. Some promising findings have resulted, which are discussed below. Furthermore, these agents are being tested in combination as all-oral regimens with and without $\mathrm{RBV}$, paving the way for interferon-free as well as RBV-free regimens.

Other novel DAAs include the polymerase inhibitors. There are two distinct groups, the nucleoside polymerase inhibitors (NIs) and nonnucleoside polymerase inhibitors (NNIs). The NIs are analogs of natural substrates that interact with the catalytic site for NS5B, incorporate into the elongating chain of HCV RNA, and subsequently cause chain termination of viral RNA synthesis. Since the active site of the polymerase is highly conserved through replication, viruses with mutations of the active site tend to lose the ability to replicate, giving the NIs a high barrier to resistance. ${ }^{14,15}$

NNIs, on the other hand, bind to several allosteric sites on the NS5B protein and change the confirmation of the active site, which in turn prevents effective viral RNA synthesis. The efficacy of antiviral activity varies by HCV genotype and subtype, and these novel drugs vary among themselves in the extent to which resistant variants develop. ${ }^{16}$

The NS5A component of the replication complex is another prime target for inhibiting HCV replication. NS5A is a multifunctional protein involved with many different stages of the HCV life cycle, including RNA replication and virion assembly and secretion. Inhibitors of the NS5A complex 
have proven to be high-potency drugs, with multigenotypic efficacy and a low-to-intermediate barrier to resistance. This effect may be especially potent when used in conjunction with another class of anti-HCV therapy. ${ }^{17}$

\section{Simeprevir}

Simeprevir (SMV), a once-daily dosed NS3/4A protease inhibitor, in combination with $\mathrm{PEG} / \mathrm{RBV}$, was approved in November 2013 by the US Food and Drug Administration (FDA) for treatment of HCV genotype 1 patients.

The PILLAR study was a randomized, placebo-controlled Phase II trial for treatment-naïve, noncirrhotic, HCV genotype 1 patients. The study showed SVR rates of $75 \%-86 \%$ in the SMV + PEG/RBV groups versus $65 \%$ in the PEG/ RBV group. For patients meeting response-guided treatment criteria, the SVR rate was $91 \%$. Discontinuation rates were $8 \%-16 \%$ in SMV-treated patients and $15 \%$ in the control group. The frequency of viral breakthrough and, consequently, of treatment cessation was $5 \%$. The majority of patients experiencing viral breakthrough or relapse had emerging NS3 protease mutations at time of failure. ${ }^{18}$

Phase III trials of SMV include QUEST-1, QUEST-2, and PROMISE. QUEST-1 and QUEST-2 randomized patients to PEG/RBV plus placebo versus SMV + PEG/RBV $150 \mathrm{mg}$ daily. Pooled data reveal overall SVR rates 12 weeks after treatment (SVR12) among HCV genotype 1 patients of $80 \%$ in the SMV + PEG/RBV group versus $50 \%$ in the placebo group. Patients with HCV genotype 1a had SVR rates of $71 \%$, versus $90 \%$ in genotype $1 \mathrm{~b}$ patients. This difference is largely due to patients with the naturally occurring $Q 80 \mathrm{~K}$ polymorphism. In fact, HCV genotype 1a patients with a Q80K mutation had SVR rates similar to those found in patients in the control arms receiving PEG/RBV (58\% and $52 \%$, respectively). Further, HCV genotype 1a patients without a $Q 80 K$ mutation matched the SVR rates of patients with HCV genotype $1 \mathrm{~b}$ (84\% versus $85 \%$ ). In QUEST-1, patients were stratified by fibrosis level and HVC subgenotype (1a versus $1 \mathrm{~b}$ ). In the SMV treatment group, patients with cirrhosis achieved an SVR rate of 58\% compared with $82 \%$ in patients without cirrhosis (Figure 1). ${ }^{19-21}$

PROMISE was a Phase III trial of treatment-experienced $\mathrm{HCV}$ genotype 1 patients who were randomized to receive SMV or placebo, each combined with PEG/RBV. This was followed by response-guided therapy for an additional 12-36 weeks of PEG/RBV. Results showed that among previous relapsers, 79\% achieved SVR12 with SMV therapy, compared to $39 \%$ in the placebo arm. In addition, the majority of patients $(93 \%)$ were eligible for shortened therapy with

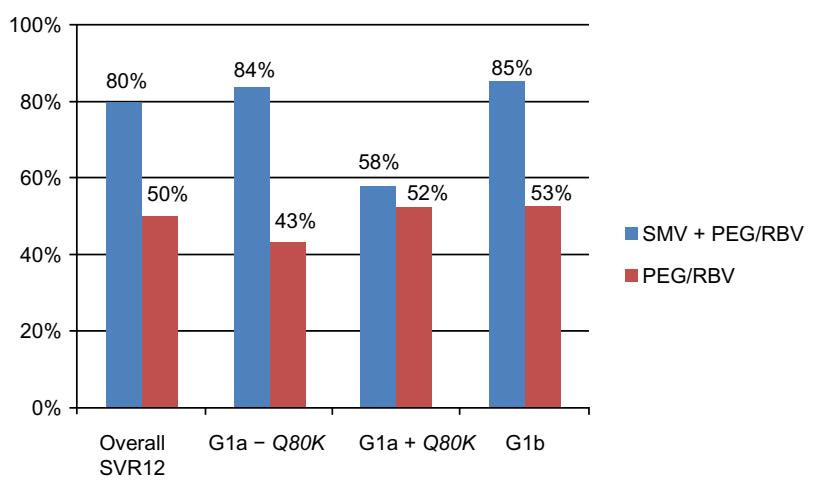

Figure I SVRI2 rates: pooled QUEST-I and QUEST-2 study results.

Notes: Randomized, double-blind, two-arm, placebo-controlled Phase III trials in 785 treatment-naive adults with HCV genotype la with and without Q80K (Gla \pm Q80K) polymorphism and patients with chronic HCV genotype Ib and compensated liver disease (including cirrhosis). Patients were treated with SMV for 12 weeks + PEG/RBV (24 or 48 weeks) versus PEG/RBV alone (48 weeks). 19,20

Abbreviations: Gla, HCV genotype la; GIb, HCV genotype Ib; HCV, hepatitis C virus; PEG, pegylated interferon- $\alpha$; $Q 80 K, Q 80 K$ polymorphism; RBV, ribavirin; SMV, simeprevir; SVR, sustained virologic response; SVRI2, sustained virologic response rate 12 weeks after treatment.

$83 \%$ SVR 12 rates. As was seen in the QUEST-1 trial, SVR 12 rates were higher in $\mathrm{HCV}$ genotype $1 \mathrm{~b}$ patients $(86 \%)$ than in genotype 1a patients (70\%). Patient subpopulations with unfavorable conditions, including those with cirrhosis or advanced fibrosis (METAVIR F4) and interleukin (IL) 28B TT genotype, also achieved higher SVR rates (74\% and $64 \%$ versus $26 \%$ and $18 \%$ respectively), when compared with placebo..$^{22}$

Adverse event profiles were similar between the SMV and placebo groups. A total of four deaths occurred in the treatment groups, but these were thought by the investigators to be unrelated to treatment. In the pooled analysis of QUEST 1, QUEST 2, and PROMISE, 2\% of those in the SMV group had serious adverse events, versus $3 \%$ of those in the control group. A total of three patients $(0.4 \%)$ in the SMV group had significant adverse events, which were determined to be related to SMV by the study investigator; one patient experienced major depression and two patients experienced photosensitivity reactions. Other common adverse events were rash $(28 \%$ in treatment groups versus $20 \%$ in control groups), influenza-like illness ( $26 \%$ in treatment groups versus $21 \%$ control groups), pruritus ( $22 \%$ in treatment groups versus $15 \%$ in control groups) and nausea (22\% in treatment groups and $18 \%$ in control groups). ${ }^{22,23}$

Overall, SMV + PEG/RBV confers similar efficacy to its DAA predecessors, BOC and TVR, with the added benefit of a more convenient dosing regimen and a more favorable side-effect profile. A meta-analysis attempted to compare the relative efficacy and safety of SMV-based therapy with BOC/ TVR-based triple therapy and concluded that SMV shows a 
similar chance of achieving an SVR with a lower probability of both the incidence of chronic HCV-related adverse events and discontinuation due to adverse events. ${ }^{24}$ It should be noted that the manufacturer of SMV has recommended, as noted in the package insert, that patients with HCV genotype 1a be tested for the $Q 80 \mathrm{~K}$ mutation prior to treatment initiation. This has raised concerns among providers, given the expense of this test and the question as to whether or not insurance providers will reimburse for this test. This may preclude some providers from considering SMV-based therapy altogether for HCV genotype 1a patients.

\section{Sofosbuvir}

Sofosbuvir (SOF) is a nucleotide analog with potent activity against $\mathrm{HCV}$ genotypes $1-6$. SOF administered in combination with RBV is the first all-oral therapy for $\mathrm{HCV}$ genotypes 2 or 3 . For patients with HCV genotypes 1, 4, 5, or $6, \mathrm{SOF}$ in combination with $\mathrm{PEG} / \mathrm{RBV}$ provides a shorter, simpler, and more effective interferon-limiting regimen.

Phase II trials showed SOF was a potent and rapid suppressor of $\mathrm{HCV}$ genotypes 1-4 and 6, with a once-daily $400 \mathrm{mg}$ dose. In addition, Phase II data revealed that HCV genotypes 2 and 3 could be efficaciously treated with SOF + RBV without PEG, while the addition of PEG to SOF + RBV treatment increased the response rate for HCV genotypes 1, 4 , and 6 and allowed the duration of therapy to be decreased to 12 weeks. ${ }^{25-27}$

Data from four Phase III trials form the principal basis for characterizing the safety and efficacy of SOF in patients with chronic $\mathrm{HCV}$ infection. Three trials were conducted in subjects with HCV genotype 2 or 3 , and one study evaluated genotypes 1, 4, 5, and 6. These trials are discussed below.

FISSION evaluated SOF + RBV treatment for 12 weeks in treatment-naïve $\mathrm{HCV}$ genotype 2 and 3 patients. The primary efficacy endpoint of this study was noninferiority of SOF + RBV alone compared to the standard 24 weeks of PEG/RBV. This endpoint was met with 67\% (170 of 253) of patients achieving SVR12 in the SOF + RBV treatment group versus $67 \%$ (162 of 243) in the PEG/RBV treatment group. Subgroup analyses were generally similar for SVR12 rates between the treatment groups for age, sex, race, ethnicity, baseline body mass index, IL28B genotype, and baseline HCV RNA. In both treatment groups, patients with HCV genotype 2 had higher SVR12 rates than did patients with genotype 3. Noncirrhotic patients also had higher SVR12 rates than did cirrhotic patients in both treatment groups (Figure 2). ${ }^{28}$

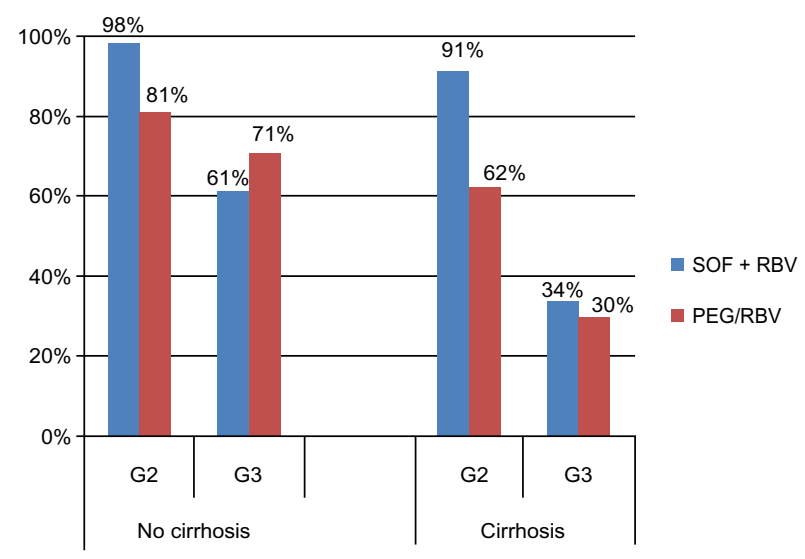

Figure 2 SVRI2 rates in treatment-naïve patients with and without cirrhosis: FISSION study results.

Notes: Comparison of SVRI2 rates in treatment-naive patients with chronic HCV genotype 2 or 3 receiving either SOF + RBV for 12 weeks or PEG/RBV for 24 weeks. ${ }^{28}$ Abbreviations: G2, HCV genotype 2; G3, HCV genotype 3; HCV, hepatitis C virus; PEG, pegylated interferon- $\alpha$; RBV, ribavirin; SOF, sofosbuvir; SVRI2, sustained virologic response rate 12 weeks after treatment.

POSITRON evaluated SOF + RBV for 12 weeks compared with placebo in patients with HCV genotype 2 or 3 who were interferon intolerant, interferon ineligible (ie, had medical conditions that precluded interferon therapy), or unwilling to take interferon. The primary efficacy analysis assessed the superiority of SOF + RBV compared with placebo. The majority of patients considered to be PEG ineligible were considered to have a psychiatric comorbidity (58\%), while most PEG intolerance was attributed to flu-like symptoms (32\%) followed by prior psychiatric sequelae with $\mathrm{PEG}$ $(20 \%)$. Overall SVR rates were $78 \%$ in combined analysis of HCV genotype 2 and 3 patients. Genotype 2 patients showed SVR rates of $93 \%$, while genotype 3 achieved SVR in only $61 \%$ of patients, although SVR rates reached as high as $70 \%$ in genotype 3 treatment-naïve patients. This reflects the favorable response of HCV genotype 2 seen in the FISSION trial. Of note, in $\mathrm{HCV}$ genotype 3 patients who underwent prior $\mathrm{HCV}$ treatment for more than 12 weeks, only $18 \%(2 / 11)$ achieved SVR. Cirrhotic patients with HCV genotype 2 had a $94 \%$ SVR rate, while cirrhotic HCV genotype 3 patients had a $21 \%$ SVR rate. ${ }^{29}$

FUSION evaluated SOF + RBV for 12 weeks or 16 weeks in treatment-experienced subjects for whom prior PEG-based therapy had failed. A SOF + RBV group with a 16-week treatment duration was included in the study to evaluate whether a longer duration would lead to improved response rates compared with a 12 -week duration in this difficultto-treat population. Overall, in this treatment-experienced population, 16 weeks of therapy proved to have significant positive impact on SVR rates. In HCV genotype 2, SVR rates 
increased from $86 \%$ to $94 \%$, and in HCV genotype 3, SVR rates increased from $30 \%$ to $62 \%$ with the 4 -weeks-longer duration of therapy. Extending treatment duration by 4 weeks conferred a significant benefit to patients with cirrhosis as well. HCV genotype 2 patients with cirrhosis saw an increase in SVR from $60 \%$ to $78 \%$, while genotype 3 patients with cirrhosis saw a marked increase in response rates, from 19\% to $61 \%$ (Figure 3 ). ${ }^{29}$

An additional trial, NEUTRINO (published with the POSITRON data), evaluated SOF + PEG/RBV for 12 weeks in HCV genotype 1, 4, 5, or 6 treatment-naïve subjects. The primary endpoint was a null SVR rate of $60 \%$ based on Phase III trials of BOC and TVR, the standard recommended regimen for this population at the time of the study. The overall SVR rate was $90 \%$. HCV genotype 1 patients achieved a rate of $89 \%$, genotype 4 patients achieved $96 \%$ SVR, a single genotype 5 patient achieved SVR, and 6 of 6 genotype 6 patients achieved SVR. Overall, 80\% (43/54) patients with cirrhosis achieved SVR. ${ }^{28}$

The frequency of any adverse event in patients receiving 12 weeks of SOF + RBV without PEG was $88 \%$, compared to $77 \%$ in the placebo group. The most common adverse event in the SOF + RBV genotype 2 and 3 pooled data was fatigue, reported in $40 \%$ of patients, followed by headache and nausea, reported in $23 \%$ and $20 \%$ of patients, respectively. The frequency of the commonly reported adverse events was similar in patients in receiving $\mathrm{SOF}+\mathrm{RBV}$ treatment for 12 and 16 weeks. ${ }^{28,29}$

Four percent of patients receiving $\mathrm{SOF}+\mathrm{RBV}$ for 12 weeks experienced a serious adverse event, while

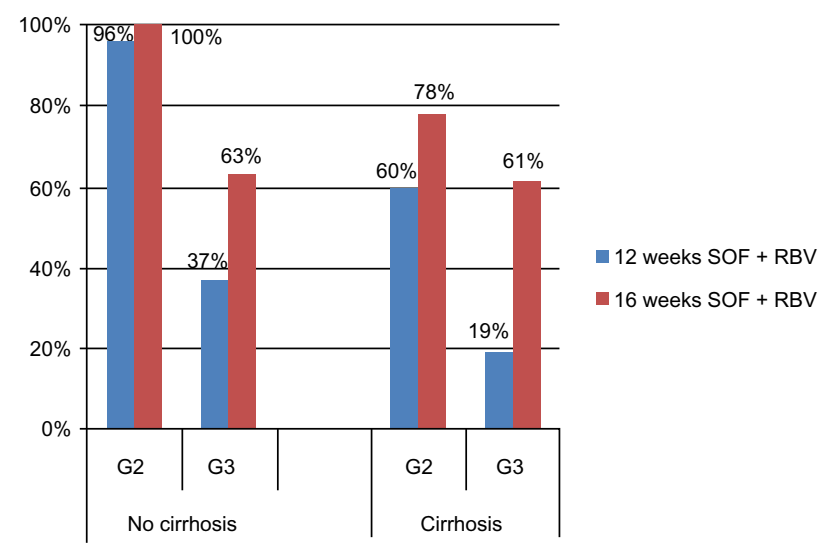

Figure 3 SVRI 2 rates in treatment-experienced patients with and without cirrhosis: FUSION study results.

Notes: Comparison of SVRI 2 in treatment-experienced patients with chronic $\mathrm{HCV}$ genotype 2 or 3 receiving SOF + RBV for either 12 weeks or 16 weeks. ${ }^{29}$ Abbreviations: G2, HCV genotype 2; G3, HCV genotype 3; HCV, hepatitis C virus; RBV, ribavirin; SOF, sofosbuvir; SVRI2, sustained virologic response rate 12 weeks after treatment.
$1 \%(8 / 566)$ patients discontinued treatment due to an adverse event. Of note, there were no treatment discontinuations in the 96 patients undergoing 16 weeks of SOF $+\mathrm{RBV}$ in the FUSION study. It should also be noted that across the four Phase III studies for SOF, no genotypic or phenotypic viral resistance to SOF or RBV was detected. The one death among all of the SOF treatment groups was determined to be caused by heroin and cocaine overdose on day 1 of treatment. ${ }^{28,29}$

With RBV monotherapy, expected hemoglobin reductions are approximately $2 \mathrm{~g} / \mathrm{dL}$. This reduction is similar to those observed in the SOF + RBV groups in the FISSION, POSITRON, and FUSION studies. In addition, SOF monotherapy was not associated with any hemoglobin reductions in the Phase II studies. In non-interferon-containing treatment groups, no effect on neutrophil or platelet counts was observed. ${ }^{26,27}$

The incidence of grade 3 total bilirubin elevation (2.5-5.0 times the upper limit of normal) in the pooled $\mathrm{SOF}+\mathrm{RBV} 12$-week treatment groups was $2 \%$. No patients with a bilirubin elevation had concomitant elevations in transaminases or clinical symptoms such as jaundice, and no patient interrupted or discontinued treatment due to elevated bilirubin. $^{29}$

Sofosbuvir is currently undergoing investigation in the pre-liver-transplant population. Infection of an uninfected, transplanted graft is universal among transplant recipients with chronic HCV. The rate of accelerated, progressive liver disease and cirrhosis in the setting of immunosuppression in the posttransplantation period is high, with rates of moderate chronic hepatitis as high as $27 \%$ after a median of 3 years and the progression to cirrhosis in $8 \%$ of patients after a median of approximately 4 years. SOF $+\mathrm{RBV}$ has been evaluated in 61 patients awaiting liver transplant to assess $\mathrm{HCV}$ recurrence posttransplant. Preliminary data reveal that, of 41 chronic $\mathrm{HCV}$ patients undergoing transplant who were given SOF + RBV while awaiting transplant, 93\% had undetectable HCV RNA at the time of transplant and $71 \%$ of patients continued to have undetectable levels 24 weeks posttransplant. ${ }^{30}$

\section{Combination all-oral therapy}

There is currently an ongoing trial of SOF + SMV with and without RBV for 12 or 24 weeks in HCV genotype 1 noncirrhotic patients who were either null-responsive to $P E G / R B V$ or treatment-naïve. Preliminary data showed that SVR rates 8 weeks after treatment (SVR8) for HCV genotypes 1a and $1 \mathrm{~b}$ were similar, and that the SVR8 rate in the treatmentexperienced cohort with 12 weeks of SOF + SMV without RBV was 93\% (13/14) (Figure 4). ${ }^{31}$ 


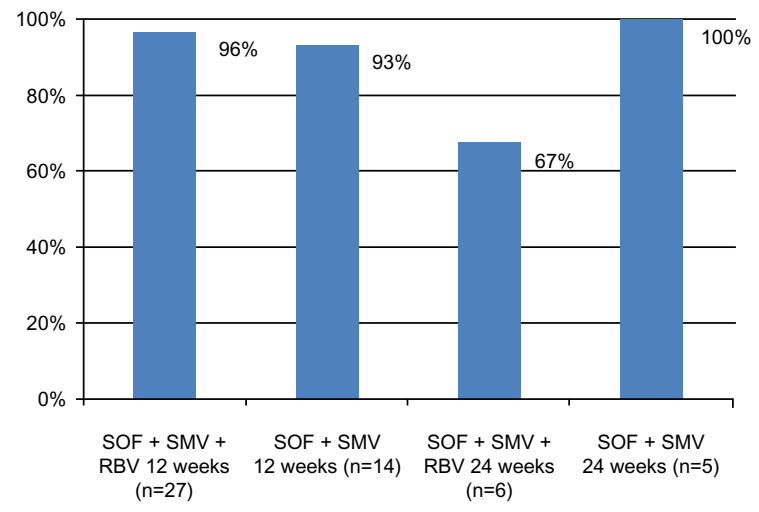

Figure 4 SVR8 rates in noncirrhotic patients with and without RBV treatment: COSMOS study results.

Notes: Results from noncirrhotic, prior null-responders to PEG/RBV with HCV genotype I receiving SOF + SMV for 12 weeks or 24 weeks, with or without RBV. ${ }^{31}$ Abbreviations: $\mathrm{HCV}$, hepatitis $\mathrm{C}$ virus; n, number; RBV, ribavirin; SMV, simeprevir; SOF, sofosbuvir; SVR8, sustained virologic response rates 8 weeks after treatment.

Daclatasvir is a first-in-class NS5A replication complex inhibitor, which has recently been tested in combination with SOF, with and without RBV, in HCV genotype 1, 2, and 3 treatment-naïve and treatment-experienced patients. Overall, 211 patients received treatment. Among patients with genotype 1 infection, $98 \%$ of naïve patients and $98 \%$ of patients with prior treatment failures achieved SVR. Ninety-two percent of patients with HCV genotype 2 and $89 \%$ of 18 patients with genotype 3 infection had a sustained virologic response at week 12. Response rates were similar among patients treated with and without RBV. HCV subtypes $1 \mathrm{a}$ and $1 \mathrm{~b}$ did not differ with respect to SVR $(98 \%$ and $100 \%$, respectively). Thirty-two patients (15\%) in this study had clinically significant fibrosis; however, a separate analysis in this population is not available. The most common adverse events were fatigue, headache, and nausea. ${ }^{32}$

Another NS5A inhibitor, ledipasvir, has been tested in combination with SOF. The LONESTAR trial evaluated $\mathrm{SOF}+$ ledipasvir with and without RBV for treatmentnaïve and treatment-experienced HCV genotype 1 patients, including patients with cirrhosis. All but two of the 100 study participants achieved SVR (Figure 5). One noncirrhotic, treatment-naïve patient in the group receiving SOF + ledipasvir for 8 weeks relapsed. The other failure was a prior treatment failure in a patient treated with 12 weeks of SOF + ledipasvir. The most common adverse events were nausea, anemia, upper respiratory tract infection, and headache..$^{33}$ A combination pill containing both SOF and ledipasvir has been developed by the manufacturer (Gilead Sciences Inc., Foster City, CA, USA), and was submitted as a New Drug Application to the FDA in February 2014. Results of this application are pending at the time of this writing.

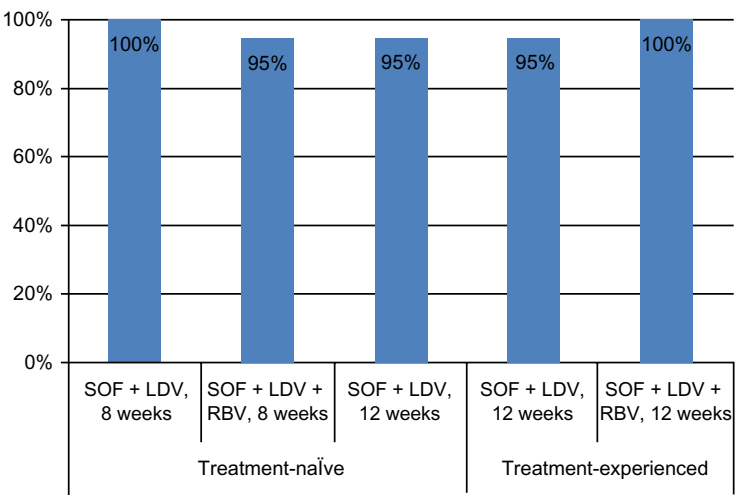

Figure 5 SVRI 2 rates in patients with HCV genotype I: LONESTAR study results. Notes: Results from treatment-naïve and treatment-experienced patients with and without cirrhosis, treated with SOF + LDV for 8 weeks or 12 weeks, with or without RBV. ${ }^{33}$

Abbreviations: HCV, hepatitis C virus; LDV, ledipasvir; RBV, ribavirin; SOF, sofosbuvir; SVRI2, sustained virologic response rate 12 weeks after treatment.

Another Phase II interferon-free trial evaluated HCV genotype 1, treatment-naïve and treatment-experienced noncirrhotic patients. This trial included a protease inhibitor, ABT-450, boosted with $100 \mathrm{mg}$ of ritonavir daily, combined with ABT-267 or ABT-333 or both, with or without RBV, for 8,12 , or 24 weeks. The rates of SVR among all treatment subgroups ranged from $83 \%-100 \%$. The rate of treatment failure was lower among those receiving three direct-acting agents plus RBV. It appeared that 12 weeks was an optimal duration of therapy, although the limit in power of the study precluded determination of statistical significance. The most frequent adverse events were fatigue, headache, nausea, and insomnia. Eight patients (1\%) discontinued treatment owing to adverse events. ${ }^{34}$

\section{Concerns about cost}

The issue of justifying the cost of new therapies has been a topic of controversy among the lay press and a concern for patients. The cost of SOF has been widely publicized, and the treatment has been critiqued for its "sticker price" of $\$ 28,000$ USD for 28 pills, such that a 12 -week course of treatment with SOF alone is more than $\$ 84,000$. Patients with compensated cirrhosis may live for more than a decade, and models suggest each individual would accrue more than $\$ 270,000$ in expenses prior to developing end-stage liver disease. ${ }^{35}$ Furthermore, according to the United Network for Organ Sharing Transplant Living Web site, the estimated US average of billed charges per liver transplant in 2011 was $\$ 577,100 . .^{36,37}$

Stratified cost-effectiveness analyses have yet to be published on our newest therapies. Even so, not only is treatment duration shortened with these therapies, but the 
costs associated with side effects due to treatment with BOC/TVR plus PEG/RBV, including blood transfusion, erythropoietin, clinic visits, and hospitalizations, are eliminated. Consequently, is it likely that the general consensus, even among insurance companies, will be that the price of treating an individual "per SVR" is actually cost-effective when factors beyond the price of the drug alone are considered.

\section{Conclusion}

The recent approval by the US FDA of the two newest DAA's, SMV and SOF, for the treatment of chronic HCV marks another treatment milestone and will likely enable many of our "warehoused" patients to now pursue or repursue a cure. Simeprevir has shown a favorable protease inhibitor profile with fewer adverse effects than its two predecessors and a shorter duration when combined with PEG/RBV. The currently approved regimen of SMV with a PEG/RBV backbone for HCV genotype 1 patients appears capable of achieving an SVR that is similar to that achieved by BOC/TVR + PEG/ $\mathrm{RBV}$, but in HCV genotype 1a patients, the possibility of the $Q 80 K$ mutation, combined with the fact that interferon is still required, may cause providers to look elsewhere for treatment options or again "shelve" their patients for future therapies. Some providers may seek preapproval from payers for off-label use of SMV in combination with SOF, which, as discussed above, has proven efficacious in a limited number of noncirrhotic, treatment-naïve and treatment-experienced, HCV genotype $1 \mathrm{a}$ and $1 \mathrm{~b}$ patients, for 12 weeks with or without RBV. SMV may find most of its usefulness in the future as a component in off-label, all-oral regimens, but it certainly remains a therapeutic option with $\mathrm{PEG} / \mathrm{RBV}$ in those well-suited (noncirrhotic, treatment-naïve, IL-28b CC, non-Q80K-mutated) HCV genotype 1 patients willing and able to tolerate PEG.

Sofosbuvir in combination with RBV has become the first all-oral, non-PEG regimen and is the first-in-its-class NS5B polymerase inhibitor to be approved for chronic HCV. While the PEG-free regimen is approved for HCV genotypes 2 and 3, its current indication for the HCV genotype 1 population still includes a PEG/RBV backbone, granted only for 12 weeks, and it brings overall SVR rates from $60 \%$ to $90 \%$. This provides a new standard of therapy and a new bar to be met for future therapies in HCV genotype 1 patients. In addition, as discussed above, numerous trials have shown that SOF in combination with other DAAs, particularly daclatasvir and ledipasvir, with and without RBV, will be extremely effective, tolerable, and durable for chronic HCV, even among difficult-to-treat patients, with unfavorable pretreatment profiles.

Further, several trials, including those with daclatasvir and ledipasvir in combination with SOF, may allow for the omission of RBV as a part of therapy. RBV-sparing regimens are another milestone in the future of chronic HCV therapy and will deliver yet another improvement in the safety and tolerability of treatment. Safety and tolerability also becomes of the utmost importance when we contemplate therapy for our fragile pretransplant population, a cohort in whom the benefit of SOF + RBV prior to transplant has shown promise in durable eradication of the virus in the posttransplant period. The clinical impact of this outcome cannot yet be quantified but will likely have significant benefit on graft survival, morbidity, and mortality for patients who receive a transplant for chronic HCV.

There are several factors whose importance and impact on care have yet to be established. It is not entirely clear if the development of resistance in previously treated patients will play a role in clinical decision making, ie, whether or not there will be a role in resistance profiling prior to treatment initiation in those for whom prior treatment has failed, as it appears our previous markers (treatment naïve/experienced, fibrosis stage, IL-28B status) will become less indicative of response to therapy.

Although tremendous strides have been made in the advancement of HCV therapy, accessibility to care and identification of those requiring treatment precludes addressing the global burden of chronic HCV. Not until the era of DAAs fully matures will widespread, "global" access to therapy be available. As such, while the new era of chronic HCV therapy remains in its adolescence, the global implication of the evolution in therapy is one that could conceivably mean eradication of hepatitis $\mathrm{C}$.

\section{Acknowledgment}

The authors acknowledge the contribution of Nancy S Reau, MD.

\section{Disclosure}

The author reports no conflicts of interest in this work.

\section{References}

1. Chak E, Talal AH, Sherman KE, Schiff ER, Saab S. Hepatitis C virus infection in USA: an estimate of true prevalence. Liver Int. 2011;31(8):1090-1101.

2. Institute of Medicine Report Brief. Hepatitis and Liver Cancer: A National Strategy for Prevention and Control of Hepatitis $B$ and C. Washington, DC: National Academy of Sciences; 2010. Available from: http://www.iom.edu/viralhepatitis. Accessed January 10, 2014. 
3. Asrani SK, Larson JJ, Yawn B, Therneau TM, Kim WR. Underestimation of liver-related mortality in the United States. Gastroenterology. 2013;145(2):375-382, e1-e2.

4. Holmberg SD, Spradling PR, Moorman AC, Denniston MM. Hepatitis C in the United States. N Engl J Med. 2013;368(20):1859-1861.

5. Ward JW, Lok AS, Thomas DL, El-Serag HB, Kim WR. Report on a single-topic conference on "Chronic viral hepatitis - strategies to improve effectiveness of screening and treatment". Hepatology. 2012;55(1):307-315.

6. Lee G, Piper DE, Wang Z, et al. Novel inhibitors of hepatitis C virus RNA-dependent RNA polymerases. J Mol Biol. 2006;357(4): 1051-1057.

7. Biswal BK, Wang M, Cherney MM, et al. Non-nucleoside inhibitors binding to hepatitis $\mathrm{C}$ virus NS5B polymerase reveal a novel mechanism of inhibition. J Mol Biol. 2006;361(1):33-45.

8. INCIVEK $^{\circledR}$ (telaprevir) [package insert]. Cambridge, MA: Vertex Pharmaceuticals Incorporated; 2013.

9. Poordad F, McCone J Jr, Bacon BR, et al; SPRINT-2 Investigators. Boceprevir for untreated chronic HCV genotype 1 infection. $N$ Engl $J$ Med. 2011;364(13):1195-1206.

10. Bacon BR, Gordon SC, Lawitz E, et al; HCV RESPOND-2 Investigators. Boceprevir for previously treated chronic HCV genotype 1 infection. N Engl J Med. 2011;364(13):1207-1217.

11. Jacobson IM, McHutchison JG, Dusheiko G, et al; ADVANCE Study Team. Telaprevir for previously untreated chronic hepatitis $\mathrm{C}$ virus infection. N Engl J Med. 2011;364(25):2405-2416.

12. Sherman KE, Flamm SL, Afdhal NH, et al. Telaprevir in combination with peginterferon alfa2a and ribavirin for 24 or 48 weeks in treatmentnaïve genotype $1 \mathrm{HCV}$ patients who achieved an extended rapid viral response: final results of phase 3 ILLUMINATE study. AASLD 2010: 61st Annual Meeting for the American Association for the Study of Liver Disease; October 29-November 2, 2010; Boston, MA. Hepatology. 2010;52(4):401A-402A.

13. Gaetano JN, Reau N. Hepatitis C: management of side effects in the era of direct-acting antivirals. Curr Gastroenterol Rep. 2013; 15(1):305

14. Lesburg CA, Cable MB, Ferrari E, Hong Z, Mannarino AF, Weber PC. Crystal structure of the RNA-dependent RNA polymerase from hepatitis C virus reveals a fully encircled active site. Nat Struct Biol. 1999;6(10):937-943.

15. Ali S, Leveque V, Le Pogam S, et al. Selected replicon variants with low-level in vitro resistance to the hepatitis C virus NS5B polymerase inhibitor PSI-6130 lack cross-resistance with R1479. Antimicrob Agents Chemother. 2008;52(12):4356-4369.

16. Powdrill MH, Bernatchez JA, Götte M. Inhibitors of the hepatitis C virus RNA-dependent RNA polymerase NS5B. Viruses. 2010;2(10):2169-2195.

17. Gao M. Antiviral activity and resistance of HCV NS5A replication complex inhibitors. Curr Opin Virol. 2013;3(5):514-520.

18. Fried MW, Buti M, Dore GJ, et al. Once-daily simeprevir (TMC435) with pegylated interferon and ribavirin in treatment-naïve genotype 1 hepatitis C: the randomized PILLAR study. Hepatology. 2013;58(6): 1918-1929.

19. Jacobson I, Dore GJ, Foster GR, et al. Simeprevir (TMC435) with peginterferon/ribavirin for chronic HCV genotype 1 infection in treatment-naïve patients: results from QUEST-1, a phase III trial. The International Liver Congress ${ }^{\mathrm{TM}}$ 2013, 48th Annual Meeting of the European Association for the Study of the Liver; April 24-28, 2013; Amsterdam, The Netherlands. J Hepatol Suppl. 2013;58(Suppl 1): S574.

20. Manns M, Marcellin P, Poordad FP, et al. Simeprevir (TMC435) with peginterferon/ribavirin for treatment of chronic HCV genotype-1 infection in treatment-nave patients: results from QUEST-2, a phase III trial. The International Liver Congress ${ }^{\mathrm{TM}}$ 2013, 48th Annual Meeting of the European Association for the Study of the Liver; April 24-28, 2013; Amsterdam, The Netherlands. J Hepatol Suppl. 2013;58(Suppl 1): S568.
21. Jacobson IM, Dore GJ, Foster GR, et al. Simeprevir (TMC435) with peginterferon/ribavirin for treatment of chronic HCV genotype 1 infection in treatment-naïve patients: efficacy in difficult-to-treat patient sub-populations in the QUEST 1 and 2 phase III trials. AASLD 2013: 64th Annual Meeting of the American Association for the Study of Liver Diseases: The Liver Meeting 2013; November 1-5, 2013; Washington, DC. Hepatology. 2013;58(S1):756A-757A.

22. Lawitz E, Forns X, Zeuzem S, et al. Simeprevir (TMC435) with peginterferon/ribavirin for treatment of chronic HCV Genotype 1 Infection in patients who relapsed after previous interferon-based therapy: results from PROMISE, a Phase III trial. Digestive Disease Week 2013; May 18-21, 2013; Orlando, FL. Gastroenterology. 2013;144(5 Suppl1): S-151.

23. Forns X, Lawitz E, Zeuzem S, et al. Simeprevir (TMC435) with peginterferon $\alpha-2 \mathrm{a} /$ ribavirin for treatment of chronic HCV genotype 1 infection in patients who relapsed after previous interferon-based therapy: efficacy and safety in patient sub-populations in the PROMISE phase III trial. AASLD 2013: 64th Annual Meeting of the American Association for the Study of Liver Diseases: The Liver Meeting 2013; November 1-5, 2013; Washington, DC. Hepatology. 2013;58(S1):737A-738A.

24. Bryden PA, Quigley JM, Padhiar A, Cerri K, Scott DA. The relative efficacy and safety of simeprevir-based triple therapy compared to boceprevir and telaprevir in treatment naïve patients chronically infected with genotype-1 hepatitis $\mathrm{C}$ virus: Bayesian network meta-analyses. AASLD 2013: 64th Annual Meeting of the American Association for the Study of Liver Diseases: The Liver Meeting 2013; November 1-5, 2013; Washington, DC. Hepatology. 2013;58(S1):756A.

25. Kowdley KV, Lawitz E, Crespo I, et al. Sofosbuvir with pegylated interferon alfa-2a and ribavirin for treatment-naive patients with hepatitis C genotype-1 infection (ATOMIC): an open-label, randomised, multicentre phase 2 trial. Lancet. 2013;381(9883):2100-2107.

26. Osinusi A, Meissner EG, Lee YJ, et al. Sofosbuvir and ribavirin for hepatitis $\mathrm{C}$ genotype 1 in patients with unfavorable treatment characteristics: a randomized clinical trial. JAMA. 2013;310(8):804-811.

27. Lawitz E, Lalezari JP, Hassanein T, et al. Sofosbuvir in combination with peginterferon alfa-2a and ribavirin for non-cirrhotic, treatment-naive patients with genotypes 1,2, and 3 hepatitis $\mathrm{C}$ infection: a randomised, double-blind, phase 2 trial. Lancet Infect Dis. 2013;13(5):401-408.

28. Lawitz E, Mangia A, Wyles D, et al. Sofosbuvir for previously untreated chronic hepatitis C infection. $N$ Engl J Med. 2013;368(20): $1878-1887$.

29. Jacobson IM, Gordon SC, Kowdley KV, et al; POSITRON Study; FUSION Study. Sofosbuvir for hepatitis C genotype 2 or 3 in patients without treatment options. N Engl J Med. 2013;368(20):1867-1877.

30. Antiviral Drugs Advisory Committee Meeting Briefing Document. Sofosbuvir for Treatment of Chronic Hepatitis C Infection. Foster City, CA: Gilead Sciences, Inc.; 2013:91-94. Available from: http://www. fda.gov/downloads/advisorycommittees/committeesmeetingmaterials/ drugs/antiviraldrugsadvisorycommittee/ucm371877.pdf. Accessed March 1, 2014.

31. Lawitz E, Ghalib R, Rodriguez-Torres M, Younossi Z, Corregidor A, Jacobson I. COSMOS Study: SVR4 results of a once-daily regimen of simeprevir (TMC435) plus sofosbuvir (GS-7977) with or without ribavirin in HCV genotype 1 null responders. 20th Conference on Retroviruses and Opportunistic Infections (CROI); March 3-6, 2013; Atlanta, GA.

32. Sulkowski MS Gardiner DF, Rodriguez-Torres M, et al; AI444040 Study Group. Daclatasvir plus sofosbuvir for previously treated or untreated chronic HCV infection. N Engl J Med. 2014;370(3):211-221.

33. Lawitz E, Poordad FF, Pang PS, et al. Sofosbuvir and ledipasvir fixeddose combination with and without ribavirin in treatment-naive and previously treated patients with genotype 1 hepatitis $C$ virus infection (LONESTAR): an open-label, randomised, phase 2 trial. Lancet. 2014;383(9916):515-523.

34. Kowdley KV, Lawitz E, Poordad F, et al. Phase $2 b$ trial of interferonfree therapy for hepatitis C virus genotype 1. N Engl J Med. 2014; 370(3):222-232. 
35. Reau NS, Jensen DM. Sticker shock and the price of new therapies for hepatitis C: Is it worth it? Hepatology. 2014.

36. Transplant Living. Costs [homepage on the Internet]. Richmond, VA: United Network for Organ Sharing; 2014. Available from: http://www. transplantliving.org/before-the-transplant/financing-a-transplant/thecosts/. Accessed February 20, 2014.
37. National Digestive Diseases Information Clearinghouse (NDDIC). Liver Transplantation [homepage on the Internet]. Bethesda, MD: National Institute of Diabetes and Digestive and Kidney Diseases. Available from: http://www.digestive.niddk.nih.gov/ddiseases/pubs/livertransplant. Accessed February 20, 2014.

\section{Publish your work in this journal}

Drug, Healthcare and Patient Safety is an international, peer-reviewed open-access journal exploring patient safety issues in the healthcare continuum from diagnostic and screening interventions through to treatment, drug therapy and surgery. The journal is characterized by the rapid reporting of reviews, original research, clinical, epidemiological and

\section{Dovepress}

post-marketing surveillance studies, risk management, health literacy and educational programs across all areas of healthcare delivery. The manuscript management system is completely online and includes a very quick and fair peer-review system. Visit http://www.dovepress.com/ testimonials.php to read real quotes from published authors.

Submit your manuscript here: http://www.dovepress.com/drug-healthcare-and-patient-safety-journal 